\title{
Dietary supplementation of finishing pigs with the docosahexaenoic acid-rich microalgae, Aurantiochytrium limacinum: effects on performance, carcass characteristics and tissue fatty acid profile
}

\author{
Colm A. Moran ${ }^{1, *}$, Mauro Morlacchini' ${ }^{2}$, Jason D. Keegan ${ }^{3}$, and Giorgio Fusconi ${ }^{2}$
}

* Corresponding Author: Colm A. Moran Tel: +33-687508761, Fax: +353-18252245,

E-mail: cmoran@alltech.com

${ }^{1}$ Regulatory Affairs Department, Alltech SARL, Rue Charles Amand, Vire 14500, France

${ }^{2}$ CERZOO S.r.I, Via Castellarino, 12 - San Bonico, Piacenza 29122, Italy

${ }^{3}$ Regulatory Affairs Department, Alltech European

Bioscience Centre, Co. Meath, A86 X006, Ireland

ORCID

Colm A. Moran

https://orcid.org/0000-0001-9135-5074

Mauro Morlacchini

https://orcid.org/0000-0002-8262-4946

Jason D. Keegan

https://orcid.org/0000-0001-8560-3772

Giorgio Fusconi

https://orcid.org/0000-0002-6986-6246

Submitted Sept 7, 2017; Revised Oct 25, 2017; Accepted Jan 18, 2018
Objective: The aim of this experiment was to evaluate the effect of dietary supplementation with the docosahexaenoic acid (DHA)-rich microalgae, Aurantiochytrium limacinum (AURA) on pig performance, carcass traits, and the fatty acid composition of pork Longissimus lumborum (LL) and backfat.

Methods: A total of 144 Pig Improvement Company (PIC) $\times$ Goland finishing pigs ( 72 females and 72 castrated males) of mean weight $117.1( \pm 13.1) \mathrm{kg}$ were blocked by sex and body weight and provided with $0 \%$ or $1 \%$ AURA in isonutritive and isocaloric diets. A total of 24 pens provided 12 replicates per treatment. Animals were weighed on day 0 and 28 with feed and water intake recorded per pen. After 31 days supplementation (28 days of study and 3 days until the slaughtering date) three animals per pen $(n=72)$ were slaughtered and the LL and backfat thickness, lean meat content and dressing percentage were recorded for the carcasses. The fatty acid (FA) profile of the LL and backfat was established by direct FA methyl ester synthesis.

Results: No differences were observed for any performance parameters or carcass traits. Supplementation with AURA resulted in significant changes to the FA profiles of both the LL and backfat with male and female pigs responding differently to supplementation in terms of particular FAs. Overall, pork LL samples had significantly higher eicosapentaenoic acid $(\mathrm{p}<0.001)$ and DHA concentrations $(\mathrm{p}<0.001)$, and higher omega-3 $(\mathrm{n}-3)$ FAs $(\mathrm{p}<0.001)$, as well as an increased omega3:omega6 $(n-3: n-6)$ ratio $(p=0.001)$. For backfat, supplementation resulted in significantly higher amounts of DHA $(\mathrm{p}<0.001)$ and $\mathrm{n}-3$ FAs $(\mathrm{p}<0.001)$.

Conclusion: These results indicate that dietary supplementation with 1\% AURA over a 31 day period can increase the FA composition of pork LL and backfat, specifically the DHA, with no major impact on growth performance and carcass traits.

Keywords: Docosahexaenoic Acid; Algae; Polyunsaturated Fatty Acids; Enrichment; Pigs

\section{INTRODUCTION}

Humans and other animals are incapable of synthesising essential fatty acids (FA) endogenously and as such, must obtain them from their diet [1]. The essential FA are split into two categories, omega 6 (n-6) and omega 3 (n-3) FA, for which linoleic acid (LA) and $\alpha$-linolenic (ALA) are the respective parent compounds [2]. The n-3 FA, in particular eicosapentaenoic acid (EPA) and docosahexaenoic acid (DHA), have been shown to have a positive impact on a variety of human health problems including cardiovascular disease and depressive disorders [3].

Oily fish are considered the best dietary source of polyunsaturated FA (PUFA), however 
the consumption of oily fish is low in western style diets [4]. In addition, the conversion of ALA into EPA and DHA is limited in the human body, and as such the direct consumption of EPA and DHA rich products is considered necessary to meet the recommended daily requirements of each [3]. The enrichment of commonly consumed products with n-3 FA represents a way to increase their consumption without requiring any drastic dietary changes. Plant and marine (algae and fish) oils have been added to animal feed in order to increase the n-3 FA content of animal products. The tissues of monogastric animals, such as pigs, are susceptible to FA alteration through dietary modification [5], making feed supplementation a viable option to increase n-3 FA concentrations in their products.

The enrichment of pork with PUFA following dietary supplementation with plant and marine (fish and algae) oils has been successfully demonstrated by a number of authors [6-11]. Vossen et al [11], recently showed that supplementation with ALA rich linseed did not increase the concentration of DHA and EPA in pork. As in humans, the conversion of ALA to long chain PUFA is limited in pigs [12]. As such, direct supplementation with DHA and EPA rich sources is required. Despite fish being considered the richest source of EPA and DHA, productivity limitations and the issue of overfishing mean that the n-3 FA requirements of the population cannot be met using fish products alone [13]. As such marine algae represent a rich source of DHA that can be produced at scale in a sustainable manner.

Supplementation with the marine algae Schizochythrum sp. has been shown to increase the levels of EPA and DHA in meat without negatively impacting the productivity of the animals $[5,10,14,15]$. Little information is available regarding dietary supplementation of pigs with other sources of algae with the potential to be heterotrophically grown, especially in low sodium conditions. Recently, the microalga, Aurantiochytrium limacinum, was fed at low inclusion concentrations, $0.25 \%$ and $0.5 \%$, to pigs for the entire grower-finisher phase, 121 days resulting in Longissimus lumborum (LL) and backfat enrichment with DHA [16,17]. However, the commercial application of algae for a prolonged feeding period may not be practical for most farms. Therefore, the aim of this study was to investigate the effect of providing a moderate level of algae supplementation for the final month prior to slaughter on the potential to enrich DHA in the LL and backfat without influencing the productivity of finishing pigs.

\section{MATERIALS AND METHODS}

\section{Animals, experimental design and diets}

The study was carried out at the CERZOO S.r.L. Research Centre (Piacenza, Italy) in compliance with G.L.P guidelines (Directives 2004/9/EC and 2004/10/EC) for the collection, handling and documentation of data. The research protocol and animal care were carried out in accordance with European guidelines on the protection of animals used for scientific purposes (Directive 2010/63/EU). A total of 144 Pig Improvement Company (PIC) $\times$ Goland finishing pigs ( 72 female and 72 castrated males) of mean weight $117.1( \pm 13.1) \mathrm{kg}$ were recruited for the study. The animals were housed in four growingfinishing rooms, with a total of 24 pens providing 12 replicates ( 6 female and 6 castrated male) for each of the two treatments. The growing-finishing rooms were equipped with a dynamic ventilation system with heating provided by water aerotherms and positive pressure ventilation achieved by variable speed fans linked to temperature sensors which automatically responded to maintain an appropriate temperature based on the age and temperature of the pigs. Pigs were fed ad libitum via a steel feeder present in each pen and were provided with free access to water. On arrival at the facility, the pigs were allowed to acclimatise for a period of seven days after which they were weighed, sorted by sex and weight and allocated to pens such that each pen contained small medium and heavy, female or castrated male pigs. The pigs were then fed the experimental diets for a period of 31 days ( 28 days study duration followed by three days until the slaughtering date).

Animals were assigned to 1 of 2 isonitrogenous and isoenergetic diets (Table 1): control diet or treatment diet supplemented with $1 \%$ heterotrophically grown unextracted $A$. limacinum (AURA; CCAP 4087/2) biomass, provided by Alltech Inc. (ALL-G-RICH, Nicholasville, KY, USA). The analytical composition of the microalgae was determined prior to the start of the study (MVTL, New Ulm, MN, USA) in compliance with current Good Laboratory Practices (GLP) guidelines: crude protein (AOAC 990.03), crude fat (AOAC 954.02), FA composition (AOAC 996.06), moisture (AOAC 930.15), and ash (AOAC 942.05). The nutrient composition of the experimental diets was established using the following standardised methods: dry matter (DM)/moisture (ISO 6496); crude protein (ISO 5983-1); crude fat (ISO 6492); crude fibre (ISO 6865); crude ash (NEN 3329; ISO 5984-2002); starch (ISO 10520); the digestible (DE) and net energies (NE) of the diets were calculated according to Noblet and Perez [18] and Noblet et al [19] respectively. DHA was determined after direct FA methyl ester synthesis according to the procedure as described by O’Fallon et al [20].

\section{Performance parameters and carcass measurements}

Pigs were individually weighed on $\mathrm{d} 0$ and $\mathrm{d} 28$ of the study. Feed and water intake was recorded for each pen from $\mathrm{d} 0$ to $\mathrm{d} 28$. Average daily gain (ADG) and gain:feed (G:F) ratio was calculated per pen. Three pigs per pen were selected based on the mean weight for the treatment group, providing a total of 72 animals for slaughter (18 female and 18 castrated males for each experimental diet). LL and backfat thicknesses were measured, where lean meat content was determined using a 
Table 1. Ingredient composition (\%) and calculated analysis of the control and docosahexaenoic acid-rich microalgae (AURA) diets

\begin{tabular}{|c|c|c|}
\hline Item & Control & $1 \%$ AURA $^{1)}$ \\
\hline \multicolumn{3}{|l|}{ Ingredient composition } \\
\hline Corn meal $(\%)$ & 55.00 & 54.95 \\
\hline Soybean meal 48\% & 13.40 & 13.10 \\
\hline Barley meal (\%) & 14.00 & 14.00 \\
\hline Wheat bran (\%) & 13.00 & 13.00 \\
\hline Hydrogenated fat of palm oil (\%) & 2.00 & 1.35 \\
\hline Dicalcium phosphate $(\%)$ & 0.40 & 0.40 \\
\hline Calcium carbonate (\%) & 1.20 & 1.20 \\
\hline L-lysine $\mathrm{HCl}$ 99\% & 0.40 & 0.40 \\
\hline Sodium chloride (\%) & 0.25 & 0.25 \\
\hline Premix grower/finisher ${ }^{2)}(\%)$ & 0.20 & 0.20 \\
\hline L-threonine (\%) & 0.12 & 0.12 \\
\hline DL-methionine 99\% & 0.03 & 0.03 \\
\hline AURA $(\%)$ & 0 & 1.00 \\
\hline \multicolumn{3}{|l|}{ Analytical characteristics } \\
\hline Dry matter (\%) & 89.92 & 89.93 \\
\hline Crude protein (\%) & 16.63 & 16.60 \\
\hline Crude fibre (\%) & 4.38 & 4.36 \\
\hline Crude fat $(\%)$ & 5.93 & 5.91 \\
\hline Starch $(\%)$ & 53.13 & 53.07 \\
\hline Total lysine (\%) & 1.08 & 1.07 \\
\hline Total methionine (\%) & 0.30 & 0.30 \\
\hline Total threonine (\%) & 0.72 & 0.71 \\
\hline Total tryptophan (\%) & 0.18 & 0.18 \\
\hline Calcium $(\%)$ & 0.72 & 0.72 \\
\hline Total phosphorus (\%) & 0.58 & 0.57 \\
\hline Sodium $(\%)$ & 0.13 & 0.13 \\
\hline Digestible energy ${ }^{3}$ (kcal/kg) & 3,713 & 3,708 \\
\hline Net energy ${ }^{3)}(\mathrm{kcal} / \mathrm{kg})$ & 2,703 & 2,705 \\
\hline
\end{tabular}

$D E$, digestible energy; $C P$, crude protein; $E E$, ether extract; $C F$, crude fibre; $N E$, net energy; ST, starch.

1) AURA, unextracted Aurantiochytrium limacinum algae containing $18.0 \mathrm{~g}$ DHA/100 g.

${ }^{2)}$ Content of vitamins and oligo minerals/kg feed: vit $A, 15,000$ IU; vit $D_{3}, 1,500$ IU; vit $E, 20$ mg; vit $B_{1}, 2$ mg; vit $B_{2}, 0.38$ mg; vit $B_{6}, 2$ mg; vit $B_{12}, 0.015$ mg; vit $\mathrm{H}, 40 \mathrm{mg}$; vit K, 2 mg; vit PP, 25 mg; vit H, 0.10 mg; D-pantothenic acid, 10 mg; choline chloride, $375 \mathrm{mg}$; manganese oxide, $60 \mathrm{mg}$; iron carbonate, $200 \mathrm{mg}$; copper sulphate, 20 mg; zinc oxide, 75 mg; Co, 0.75 mg; potassium iodide, 2.4 $\mathrm{mg}$; sodium selenite, $0.30 \mathrm{mg}$.

${ }^{3)}$ The digestible energies of the diets were calculated according to Noblet and Perez [18] using the following equation: $D E=4,151-(122 \times \%$ ash $)+(2$ $3.2 \times \% \mathrm{CP})+(38.2 \times \% \mathrm{EE})-(64.2 \times \% \mathrm{CF})$. The net energies of the diets were calculated according to Noblet et al [19] using the following equation: $\mathrm{NE}=$ $2,796+4.15 \times \mathrm{EE}+0.81 \times \mathrm{ST}-7.07 \times$ ash $-5.38 \times \mathrm{CF}$.

Fat-O-Meter (SFK Technology, Herlev, Denmark) optical probe positioned $8 \mathrm{~cm}$ to the side of the central line of the carcass between the third and fourth last ribs, in accordance with the Commission Implementing Decision of 24 January 2014 on authorizing methods for grading pig carcasses in Italy (2014/ 38/EU). Carcasses were classified as per the SEUROP carcass classification system (Reg. 2013/1308/EU). LL muscle and backfat Samples were removed from the right side of each animal, within 15 min post-slaughter, between the third and fourth last ribs, placed in vacuum bags and immediately frozen and stored at $-18^{\circ} \mathrm{C}$ until FA analysis.

The FA profiles of carcass samples were determined at the Institute of Food Science and Nutrition (Faculty of Agricultural Sciences, Food and Environment, Catholic University of Sacred Heart, Piacenza, Italy). Samples were thawed, ground and homogenized for subsequent FA profile analysis after direct FA methyl ester synthesis according to the procedure as described by O'Fallon et al [20]. The complete FA profiles included short-chain FAs (C4.0 through C8.0), medium-chain FAs (C10.0 through C15.1), long-chain FAs (C16.0 through C22:6n3), and very long-chain FA (C24:0 and C24:1). The total omega-3 FA composition was calculated as the $\sum$ ( $\alpha$-linolenic acid [C18:3n3]+cis-11,14,17-eicosatrienoic acid [C20:3n3]+ EPA [C20:5n3]+DHA [C22:6n3]). The total omega-6 FA composition was calculated as the $\sum$ (linolelaidic acid [C18:2n6trans] + linoleic acid [C18:2n6cis] $+\gamma$-linolenic acid [C18:3n6]+ cis-8,11,14-eicosatrienoic acid [C20:3n6]+arachidonic acid [C20:4n6]).

\section{Statistical analysis}

Data were analysed by the general linear model (GLM) procedure of SAS (2010, release 9.3) using analysis of variance (ANOVA) as the main statistical test. For the performance parameters the pen was the experimental unit with 24 replicates blocked by sex (12 castrated male pens and 12 female pens). For each parameter the GLM was fitted with the following terms: Treatment (Control, AURA); Sex (Castrated male, female); plus the treatment $\times$ sex interaction. For the carcass characteristics and the FA profile the pig was the experimental unit with a total of 72 replicates ( 36 castrated male, 36 female). When a significant treatment $\times$ sex interaction was detected, the sexes were analysed separately by GLM to investigate the effect of treatment on either castrated male or female pigs. Students " $\mathrm{t}$ " and Tukey tests were used to compare the means of each group. For the ANOVA model, $\mathrm{p} \leq 0.05$ indicated a significant difference while $0.05<\mathrm{p} \leq 0.10$ indicated a trend.

\section{RESULTS}

Diet analysis, performance and carcass characteristics The test article, AURA, used in the study primarily consisted of $70.2 \mathrm{~g}$ crude fat $/ 100 \mathrm{~g}$ DM biomass composed of a significant level of palmitic acid and DHA, $34.5 \mathrm{~g}$ and $18.0 \mathrm{~g} / 100 \mathrm{~g}$ DM biomass respectively. Additionally, AURA contained $15.8 \%$ crude protein, $3.9 \%$ ash, and $2.3 \%$ moisture. The EPA accounted for only $0.23 \mathrm{~g} / 100 \mathrm{~g}$ DM biomass. The chemical composition, energy value and DHA content of the experimental diets are show in Table 2. The pigs maintained good health throughout the study with no animals being removed or culled for welfare reasons. Treatment with AURA had no effect on ADG, average daily feed intake, average daily water 
Table 2. Analytical characteristics of the finishing pig experimental diets on a dry matter basis

\begin{tabular}{lcc}
\hline Item & Control & 1\% AURA \\
\hline Dry matter (\%) & $89.22 \pm 0.18$ & $89.37 \pm 0.16$ \\
Crude protein (\%) & $16.39 \pm 0.13$ & $16.53 \pm 0.08$ \\
Crude fibre (\%) & $4.22 \pm 0.09$ & $4.11 \pm 0.02$ \\
Crude fat (\%) & $5.77 \pm 0.13$ & $5.78 \pm 0.04$ \\
Starch (\%) & $51.99 \pm 0.21$ & $52.46 \pm 0.03$ \\
Digestible energy ${ }^{2)}(\mathrm{kcal} / \mathrm{kg})$ & $2,978 \pm 20$ & $3,024 \pm 21$ \\
Net energy ${ }^{2)}(\mathrm{kcal} / \mathrm{kg})$ & $2,503 \pm 5$ & $2,510 \pm 4$ \\
DHA (g/kg) & 0 & $1.62 \pm 0.08$ \\
\hline DHA &
\end{tabular}

$D H A$, docosahexaenoic acid; $D E$, digestible energy; $C P$, crude protein; $E E$, ether extract; CF, crude fibre; NE, net energy; ST, starch.

1) AURA, unextracted Aurantiochytrium limacinum algae containing $18.0 \mathrm{~g}$ DHA/100 g.

${ }^{2)}$ The digestible energies of the diets were calculated according to Noblet and Perez [18] using the following equation: $D E=4,151-(122 \times \%$ ash $)+(2$ $3.2 \times \% \mathrm{CP})+(38.2 \times \% \mathrm{EE})-(64.2 \times \% \mathrm{CF})$. The net energies of the diets were calculated according to Noblet et al [19] using the following equation: $\mathrm{NE}=$ $2,796+4.15 \times \mathrm{EE}+0.81 \times \mathrm{ST}-7.07 \times$ Ash $-5.38 \times \mathrm{CF}$

intake, or G:F ratio (Table 3). No differences were observed between the groups in terms of LL thickness, backfat thickness, lean meat content or dressing out (Table 3). Carcass evaluation according to the SEUROP system is provided in Table 4 and indicates the expected difference in lean meat percentage between the sexes.

\section{Fatty acid profile}

Supplementation with AURA resulted in significantly higher levels of the following FAs in the pork LL samples: C8:0, C10:0, C14:0, C15:0, C16:0, C18:0, C18:1n9 cis, C18:2n6c, C18:3n3, C20:2, C22:1n9, C24:0, EPA, and DHA (Table 5). Animals supplemented with AURA had higher PUFA, n-3, and n-6 FAs,
Table 4. Effect of supplementation of a finisher pig diet for 31 days with a docosahexaenoic acid rich microalgae (AURA) at 1\% on EUROP carcass classification $^{11}$

\begin{tabular}{|c|c|c|c|c|c|}
\hline \multirow{2}{*}{ EUROP score } & \multirow{2}{*}{$\begin{array}{l}\text { Lean meat } \\
\text { content }(\%)\end{array}$} & \multicolumn{2}{|c|}{ Control } & \multicolumn{2}{|c|}{$1 \%$ AURA $^{2)}$} \\
\hline & & $n$ & $\%$ & $\mathrm{n}$ & $\%$ \\
\hline \multicolumn{6}{|c|}{ All animals $(n=36)$} \\
\hline S & $\geq 60$ & 0 & 0 & 0 & 0 \\
\hline$E$ & $55-60$ & 5 & 14 & 7 & 20 \\
\hline$U$ & $50-55$ & 18 & 50 & 18 & 50 \\
\hline$R$ & $45-50$ & 11 & 30 & 8 & 22 \\
\hline 0 & $40-45$ & 1 & 3 & 3 & 8 \\
\hline $\mathrm{P}$ & $<40$ & 1 & 3 & 0 & 0 \\
\hline \multicolumn{6}{|c|}{ Females $(n=18)$} \\
\hline$S$ & $\geq 60$ & 0 & 0 & 0 & 0 \\
\hline$E$ & $55-60$ & 5 & 28 & 5 & 28 \\
\hline U & $50-55$ & 8 & 44 & 8 & 44 \\
\hline$R$ & $45-50$ & 5 & 28 & 3 & 17 \\
\hline 0 & $40-45$ & 0 & 0 & 2 & 11 \\
\hline$P$ & $<40$ & 0 & 0 & 0 & 0 \\
\hline \multicolumn{6}{|l|}{ Males $(n=18)$} \\
\hline S & $\geq 60$ & 0 & 0 & 0 & 0 \\
\hline$E$ & $55-60$ & 0 & 0 & 2 & 11 \\
\hline$U$ & $50-55$ & 10 & 55 & 10 & 55 \\
\hline$R$ & $45-50$ & 6 & 33 & 5 & 28 \\
\hline 0 & $40-45$ & 1 & 6 & 1 & 6 \\
\hline$P$ & $<40$ & 1 & 6 & 0 & 0 \\
\hline
\end{tabular}

1) EUROP classification, Regulation 2013/1308/EU.

${ }^{2)}$ AURA, unextracted Aurantiochytrium limacinum algae containing $18.0 \mathrm{~g}$ DHA/100 g.

in addition to an increased n-3:n-6 ratio. The GLM results indicated an interaction between sex and treatment for some FAs and as such the male and female pigs were analysed separately to explore the sex specific effects. The FA profile of the

Table 3. Effect of supplementation with a docosahexaenoic-rich microalgae (AURA) at $1 \%$ of diet on performance parameters and carcass characteristics of finishing pigs

\begin{tabular}{|c|c|c|c|c|c|c|}
\hline \multirow{2}{*}{ Item } & \multirow{2}{*}{ Control } & \multirow{2}{*}{$1 \%$ AURA $^{1)}$} & \multirow{2}{*}{ Standard error } & \multicolumn{3}{|c|}{ p-value } \\
\hline & & & & Treatment effect ${ }^{2)}$ & Sex effect ${ }^{2)}$ & T×S interaction ${ }^{2)}$ \\
\hline \multicolumn{7}{|l|}{ Performance parameters ${ }^{3)}$} \\
\hline Weight d $28(\mathrm{~kg})$ & 141.2 & 140.3 & 2.89 & 0.75 & 0.11 & 0.82 \\
\hline Average daily gain (g) & 838.3 & 847.7 & 40.5 & 0.82 & 0.73 & 0.37 \\
\hline Daily feed intake (kg) & 3.405 & 3.392 & 0.02 & 0.51 & 0.56 & 0.70 \\
\hline G:F ratio & 4.10 & 4.05 & 0.01 & 0.74 & 0.66 & 0.48 \\
\hline \multicolumn{7}{|l|}{ Carcass characteristics $^{4)}$} \\
\hline Backfat thickness (mm) & 30.31 & 30.33 & 1.53 & 0.99 & 0.09 & 0.57 \\
\hline Longissimus lumborum thickness (mm) & 51.86 & 50.14 & 2.42 & 0.48 & 0.33 & 0.27 \\
\hline Lean meat content $(\%)$ & 51.00 & 50.80 & 0.91 & 0.82 & 0.06 & 0.40 \\
\hline Dressing $(\%)$ & 76.92 & 76.59 & 0.43 & 0.59 & 0.17 & 0.08 \\
\hline
\end{tabular}


Table 5. Pork Longissimus lumborum (LL) fatty acid (FA) profile (mg/100 g tissue) for control and AURA') supplemented pigs

\begin{tabular}{|c|c|c|c|c|c|c|c|c|c|c|c|c|c|}
\hline \multirow{2}{*}{ Fatty acid ${ }^{2)}$} & \multicolumn{5}{|c|}{ All pigs } & \multicolumn{4}{|c|}{ Female pigs } & \multicolumn{4}{|c|}{ Male pigs } \\
\hline & Control & AURA & SEM & Treat $^{3)}$ & $\mathrm{T} \times \mathrm{S}^{3)}$ & Control & AURA & SEM & Treat & Control & AURA & SEM & Treat \\
\hline$C 8: 0$ & 1.0 & 1.3 & 0.1 & 0.003 & 0.094 & 0.9 & 1.3 & 0.1 & 0.019 & 1.2 & 1.4 & 0.1 & 0.065 \\
\hline C10:0 & 10.3 & 12.8 & 0.6 & 0.005 & 0.079 & 9.2 & 12.0 & 0.8 & 0.021 & 11.4 & 13.5 & 0.9 & 0.092 \\
\hline C12:0 & 13.3 & 15.0 & 0.7 & 0.113 & 0.091 & 11.7 & 14.7 & 1.0 & 0.040 & 14.9 & 15.3 & 1.1 & 0.813 \\
\hline C14:0 & 156.1 & 183.3 & 7.9 & 0.017 & 0.071 & 138.2 & 178.5 & 11.0 & 0.014 & 174.0 & 188.2 & 11.3 & 0.382 \\
\hline C14:1 & 1.9 & 2.2 & 0.1 & 0.092 & 0.032 & 1.6 & 2.0 & 0.2 & 0.083 & 2.2 & 2.3 & 0.1 & 0.564 \\
\hline C15:0 & 6.0 & 7.0 & 0.3 & 0.033 & 0.602 & 5.7 & 7.3 & 0.5 & 0.040 & 6.2 & 6.8 & 0.5 & 0.384 \\
\hline C16:0 & $2,380.4$ & $2,765.3$ & 99.9 & 0.008 & 0.038 & $2,131.9$ & $2,683.1$ & 131.3 & 0.006 & $2,628.9$ & $2,847.5$ & 150.6 & 0.312 \\
\hline C16:1 & 216.7 & 245.7 & 11.7 & 0.083 & 0.043 & 190.3 & 231.4 & 16.7 & 0.092 & 243.0 & 260.1 & 16.3 & 0.463 \\
\hline C17:0 & 28.1 & 32.3 & 1.5 & 0.054 & 0.963 & 27.7 & 32.2 & 2.1 & 0.148 & 28.5 & 32.4 & 2.1 & 0.205 \\
\hline C18:0 & 1078 & $1,244.8$ & 43.1 & 0.008 & 0.197 & 1005 & $1,215.2$ & 55.4 & 0.011 & 1151 & $1,274.3$ & 66.1 & 0.196 \\
\hline C18:1 trans & 19.7 & 18.3 & 0.6 & 0.086 & 0.798 & 20.1 & 18.1 & 0.8 & 0.098 & 19.4 & 18.5 & 0.8 & 0.445 \\
\hline C18:1n9 cis & $3,592.8$ & $4,234.4$ & 152.4 & 0.004 & 0.063 & $3,249.1$ & $4,109.4$ & 188.0 & 0.003 & $3,936.5$ & $4,359.4$ & 239.8 & 0.221 \\
\hline C18:1 cis11 & 269.5 & 285.3 & 9.9 & 0.267 & 0.068 & 248.0 & 276.0 & 12.5 & 0.122 & 291.1 & 294.5 & 15.4 & 0.878 \\
\hline C18:2n6c & $1,131.6$ & $1,314.8$ & 48.9 & 0.010 & 0.185 & $1,058.8$ & $1,369.5$ & 68.7 & 0.003 & $1,204.3$ & $1,260.1$ & 69.7 & 0.575 \\
\hline C18:3n3 (ALA) & 49.6 & 59.3 & 2.5 & 0.007 & 0.182 & 45.1 & 60.5 & 3.5 & 0.004 & 54.1 & 58.2 & 3.5 & 0.415 \\
\hline C18:3n6 & 3.0 & 3.3 & 0.1 & 0.203 & 0.364 & 3.1 & 3. & 0.2 & 0.182 & 3.0 & 3.1 & 0.2 & 0.782 \\
\hline C20:0 & 8.4 & 9.4 & 0.4 & 0.065 & 0.015 & 7.4 & 9.1 & 0.4 & 0.010 & 9.5 & 9.7 & 0.6 & 0.764 \\
\hline C20:1 & 59.5 & 64.7 & 2.6 & 0.164 & 0.010 & 51.6 & 62.5 & 2.7 & 0.008 & 67.3 & 66.8 & 4.4 & 0.930 \\
\hline$C 20: 2$ & 42.8 & 48.0 & 1.9 & 0.050 & 0.125 & 39.3 & 49.6 & 2.2 & 0.002 & 46.3 & 46.5 & 3.0 & 0.963 \\
\hline C20:3n3 & 59.9 & 58.9 & 0.8 & 0.360 & 0.000 & 62.1 & 61.6 & 1.1 & 0.761 & 57.7 & 56.2 & 1.0 & 0.314 \\
\hline C20:3n6 & 13.7 & 14.3 & 0.4 & 0.254 & 0.534 & 13.4 & 14.7 & 0.5 & 0.092 & 14.0 & 14.0 & 0.6 & 0.985 \\
\hline $\mathrm{C} 20: 4 \mathrm{n} 6$ & 10.0 & 11.3 & 0.5 & 0.050 & 0.054 & 8.8 & 11.4 & 0.6 & 0.003 & 11.1 & 11.2 & 0.8 & 0.955 \\
\hline C20:5n3 (EPA) & 2.1 & 3.4 & 0.1 & $<0.001$ & 0.144 & 2.0 & 3.2 & 0.1 & $<0.001$ & 2.2 & 3.5 & 0.1 & $<0.001$ \\
\hline C22:1n9 & 1.2 & 1.6 & 0.1 & 0.000 & 0.153 & 1.1 & 1.6 & 0.1 & 0.002 & 1.4 & 1.0 & 0.1 & 0.056 \\
\hline C22:6n3 (DHA) & 9.0 & 31.2 & 0.9 & $<0.001$ & 0.803 & 9.2 & 31.7 & 1.4 & $<0.001$ & 8.8 & 30.6 & 1.1 & $<0.001$ \\
\hline $\mathrm{C} 24: 0$ & 12.9 & 11.7 & 0.3 & 0.015 & 0.276 & 12.9 & 12.2 & 0.4 & 0.324 & 12.9 & 11.1 & 0.5 & 0.020 \\
\hline$\Sigma$ Short (chain) & 1.0 & 1.3 & 0.1 & 0.003 & 0.094 & 0.9 & 1.3 & 0.1 & 0.019 & 1.2 & 1.4 & 0.1 & 0.065 \\
\hline$\Sigma$ Medium chain) & 187.6 & 220.3 & 9.5 & 0.018 & 0.079 & 166.5 & 214.5 & 13.3 & 0.015 & 208.7 & 226.1 & 13.6 & 0.375 \\
\hline$\Sigma$ Long chain ${ }^{4)}$ & $8,989.1$ & $10,458.1$ & 353.9 & 0.005 & 0.072 & 8187 & $10,256.6$ & 449.0 & 0.003 & $9,791.2$ & $10,659.5$ & 547.1 & 0.270 \\
\hline$\Sigma$ SSaturated FA & $3,694.6$ & $4,282.9$ & 149.6 & 0.007 & 0.059 & $3,350.8$ & $4,165.5$ & 194.0 & 0.005 & $4,038.5$ & $4,400.2$ & 227.8 & 0.269 \\
\hline$\Sigma$ Unsaturated FA & $5,483.1$ & $6,396.8$ & 219.9 & 0.005 & 0.093 & $5,003.7$ & $6,306.9$ & 280.1 & 0.002 & $5,962.5$ & $6,486.7$ & 339.0 & 0.282 \\
\hline$\Sigma$ Monounsaturated FA & $4,161.4$ & $4,852.2$ & 173.5 & 0.006 & 0.055 & $3,761.9$ & $4,701.1$ & 216.6 & 0.004 & $4,560.9$ & $5,003.2$ & 271.2 & 0.257 \\
\hline ¿Polyunsaturated FA & $1,321.7$ & $1,544.6$ & 54.8 & 0.005 & 0.193 & $1,241.8$ & $1,605.7$ & 76.3 & 0.002 & $1,401.6$ & $1,483.5$ & 78.7 & 0.467 \\
\hline Omega $3^{5)}$ & 120.7 & 152.8 & 3.5 & $<0.001$ & 0.395 & 118.5 & 157.1 & 4.8 & $<0.001$ & 122.8 & 148.5 & 5.1 & 0.001 \\
\hline Omega $6^{6)}$ & $1,158.3$ & $1,343.8$ & 49.7 & 0.010 & 0.185 & $1,084.1$ & $1,399.1$ & 69.7 & 0.003 & $1,232.5$ & $1,288.4$ & 71.0 & 0.581 \\
\hline Omega 3/0mega 6 & 0.106 & 0.117 & 0.0023 & 0.001 & 0.094 & 0.111 & 0.116 & 0.003 & 0.358 & 0.102 & 0.119 & 0.003 & $<0.001$ \\
\hline
\end{tabular}

SEM, standard Error of the mean; ALA, a-linolenic acids; EPA, eicosapentaenoic acid; DHA, docosahexaenoic acid; FA, fatty acids; MUFA, monounsaturated fatty acid; PUFA, polyunsaturated fatty acids.

1) AURA, unextracted Aurantiochytrium limacinum algae containing $18.0 \mathrm{~g} \mathrm{DHA} / 100 \mathrm{~g}$.

2) FA below the level of detection are not reported and include the following: C4:0, C6:0, C11:0, C13:0, C:15:1, C17:1, C18:2n6t, C18:2 c9,t11-CLA, C19:0, C21:0, C22:0, C22:C24:1

3) Effect of treatment and the treatment $\times$ sex interaction reported from the general linear model investigating the effect of treatment on fatty acid profile.

4) Short-chain fatty acids: C4.0 to C8.0; medium-chain fatty acids: C10.0 to C15.1; long-chain fatty acids: C16.0 to C22:6n3.

${ }^{5)}$ The total omega-3 fatty acid composition was calculated as the $\sum$ (a-linolenic acid [C18:3n3]+cis-11,14,17-eicosatrienoic acid [C20:3n3]+EPA [C20:5n3]+DHA [C22:6n3]).

${ }^{6)}$ The total omega-6 fatty-acid composition was calculated as the $\sum$ (linolelaidic acid [C18:2n6trans]+linoleic acid [C18:2n6cis] $+\gamma$-linolenic acid [C18:3n6]+cis-8,11,14-eicosatrienoic acid [C20:3n6]+arachidonic acid [C20:4n6]).

AURA supplemented female pigs showed increased concentrations of the following FAs: C8:0, C10:0, C12:0, C14:0, C15:0, C16:0, C18:0, C18:1n9 cis, C18:2n6c, C18:3n3, C20:0, C20:1, C20:2, C20:4n6, C22:1n9, EPA, and DHA. In contrast, the FA profile of the AURA supplemented male pigs differed only in terms of a higher EPA and DHA content and a lower C24:0 content in the LL meat. Both male and female pigs had significantly higher n-3 FA levels following AURA supplementation. Female pigs had a greater $\mathrm{n}-6$ content while male pigs did not. As such, the n-3:n-6 ratio for the female pigs did not differ between control and supplemented groups while and increase in the ratio was observed for the male pigs (Table 6). 
Table 6. Pork backfat fatty acid profile (mg/100 g tissue) for control and AURA supplemented pigs

\begin{tabular}{|c|c|c|c|c|c|c|c|c|c|c|c|c|c|}
\hline \multirow{2}{*}{ Fatty acid ${ }^{1)}$} & \multicolumn{5}{|c|}{ All pigs } & \multicolumn{4}{|c|}{ Female } & \multicolumn{4}{|c|}{ Male } \\
\hline & Control & AURA $^{2)}$ & SEM & Treat $^{3)}$ & $\mathrm{T} \times \mathrm{S}^{3)}$ & Control & AURA & SEM & Treat & Control & AURA & SEM & Treat \\
\hline$C 8: 0$ & 4.4 & 4.5 & 0.1 & 0.848 & 0.350 & 4.4 & 4.3 & 0.1 & 0.646 & 4.4 & 4.6 & 0.1 & 0.452 \\
\hline $\mathrm{C} 10: 0$ & 45.8 & 47.5 & 0.8 & 0.163 & 0.018 & 45.3 & 45.1 & 1.3 & 0.916 & 46.4 & 49.9 & 1.1 & 0.032 \\
\hline $\mathrm{C} 12: 0$ & 109.4 & 105.6 & 3.0 & 0.373 & 0.648 & 112.2 & 105.8 & 3.9 & 0.253 & 106.6 & 105.3 & 4.6 & 0.848 \\
\hline C14:0 & $1,074.9$ & $1,075.5$ & 18.2 & 0.981 & 0.911 & $1,081.1$ & $1,070.7$ & 28.5 & 0.797 & $1,068.6$ & $1,080.3$ & 22.7 & 0.718 \\
\hline C14:1 & 15.0 & 15.2 & 0.4 & 0.771 & 0.024 & 14.4 & 14.3 & 0.5 & 0.987 & 15.7 & 16.0 & 0.6 & 0.681 \\
\hline C15:0 & 57.5 & 61.0 & 2.0 & 0.211 & 0.069 & 62.1 & 62.1 & 3.4 & 0.990 & 52.9 & 60.0 & 2.1 & 0.024 \\
\hline C16:0 & $16,285.9$ & $16,422.6$ & 225.7 & 0.670 & 0.970 & $16,274.5$ & $16,367.6$ & 349.3 & 0.852 & $16,297.2$ & $16,477.5$ & 286.1 & 0.659 \\
\hline C16:1 & $1,376.8$ & $1,374.3$ & 35.4 & 0.960 & 0.276 & 1,338.8 & $1,331.3$ & 48.6 & 0.914 & $1,414.9$ & $1,417.4$ & 51.6 & 0.973 \\
\hline C17:0 & 297.0 & 306.1 & 11.4 & 0.576 & 0.028 & 328.3 & 306.1 & 19.1 & 0.418 & 265.7 & 306.1 & 12.5 & 0.028 \\
\hline C18:0 & 7,993.5 & $8,150.9$ & 181.8 & 0.542 & 0.634 & $8,158.5$ & $8,206.8$ & 254.3 & 0.894 & $7,828.4$ & $8,095.1$ & 259.9 & 0.473 \\
\hline C18:1 trans & 188.5 & 169.9 & 4.2 & 0.003 & 0.001 & 202.9 & 178.8 & 6.4 & 0.012 & 174.1 & 160.9 & 5.4 & 0.097 \\
\hline C18:1n9 cis & $27,616.9$ & $27,488.3$ & 352.8 & 0.798 & 0.568 & $27,955.3$ & $27,654.4$ & 513.4 & 0.681 & $27,278.5$ & $27,322.3$ & 484.2 & 0.949 \\
\hline C18:1 cis11 & $1,868.1$ & $1,851.4$ & 29.8 & 0.693 & 0.895 & $1,859.4$ & $1,840.4$ & 42.3 & 0.753 & $1,876.9$ & 1862.4 & 42.0 & 0.809 \\
\hline $\mathrm{C} 18: 2 \mathrm{n} 6 \mathrm{c}$ & $10,505.8$ & $10,471.5$ & 174.2 & 0.890 & 0.040 & $10,771.9$ & $10,838.3$ & 255.7 & 0.855 & $10,239.7$ & $10,104.7$ & 236.6 & 0.689 \\
\hline C18:3n3 (ALA) & 483.0 & 485.4 & 8.3 & 0.839 & 0.577 & 488.4 & 492.2 & 12.2 & 0.823 & 477.6 & 478.5 & 11.3 & 0.955 \\
\hline $\mathrm{C} 18: 3 \mathrm{n} 6$ & 20.9 & 19.9 & 0.5 & 0.166 & 0.052 & 22.0 & 20.7 & 0.7 & 0.228 & 19.9 & 19.1 & 0.8 & 0.446 \\
\hline $\mathrm{C} 20: 0$ & 94.3 & 100.4 & 2.7 & 0.119 & 0.810 & 92.7 & 101.3 & 3.9 & 0.129 & 95.8 & 99.4 & 3.8 & 0.504 \\
\hline $\mathrm{C} 20: 1$ & 513.1 & 506.1 & 11.3 & 0.661 & 0.783 & 505.5 & 508.2 & 12.1 & 0.874 & 520.7 & 503.9 & 19.1 & 0.538 \\
\hline$C 20: 2$ & 422.7 & 428.5 & 12.3 & 0.739 & 0.304 & 417.0 & 446.9 & 19.5 & 0.287 & 428.3 & 410.2 & 15.1 & 0.400 \\
\hline$C 20: 3 n 3$ & 151.2 & 146.9 & 3.0 & 0.311 & $<0.001$ & 163.3 & 155.5 & 4.7 & 0.2 & 139.2 & 138.4 & 3.8 & 0.877 \\
\hline$C 20: 3 n 6$ & 76.8 & 73.4 & 1.7 & 0.163 & 0.023 & 79.8 & 77.2 & 2.3 & 0.413 & 73.8 & 69.6 & 2.5 & 0.527 \\
\hline $\mathrm{C} 20: 4 \mathrm{n} 6$ & 97.8 & 95.8 & 2.0 & 0.489 & 0.314 & 97.5 & 98.8 & 2.5 & 0.706 & 98.0 & 92.8 & 3.1 & 0.240 \\
\hline C20:5n3 (EPA) & 0.0 & 0.0 & 0.0 & n/a & $\mathrm{n} / \mathrm{a}$ & 0.0 & 0.0 & $\mathrm{n} / \mathrm{a}$ & $\mathrm{n} / \mathrm{a}$ & 0.0 & 0.0 & $\mathrm{n} / \mathrm{a}$ & n/a \\
\hline C22:1n9 & 11.3 & 13.4 & 0.4 & 0.002 & 0.692 & 11.6 & 13.1 & 0.7 & 0.116 & 11.1 & 13.6 & 0.6 & 0.003 \\
\hline C22:6n3 (DHA) & 61.6 & 192.4 & 6.6 & $<0.001$ & 0.986 & 62.6 & 191.8 & 10.9 & $<0.001$ & 60.6 & 192.9 & 7.3 & $<0.001$ \\
\hline $\mathrm{C} 24: 0$ & 57.6 & 53.6 & 1.4 & 0.043 & 0.023 & 60.7 & 56.0 & 1.8 & 0.067 & 54.6 & 51.1 & 2.2 & 0.271 \\
\hline$\sum$ Short chain & 4.4 & 4.5 & 0.1 & 0.848 & 0.350 & 4.4 & 4.3 & 0.1 & 0.646 & 4.4 & 4.6 & 0.1 & 0.452 \\
\hline$\Sigma$ Medium chain ${ }^{4)}$ & $1,302.6$ & $1,304.8$ & 22.4 & 0.949 & 0.820 & 1,315 & 1,298 & 34.9 & 0.733 & 1290.1 & $1,311.5$ & 28.0 & 0.593 \\
\hline ¿Long chain ${ }^{4)}$ & $68,122.8$ & $68,350.7$ & 783.0 & 0.838 & 0.493 & $68,890.8$ & $68,885.5$ & 1141.1 & 0.997 & $67,354.8$ & 67,816 & $1,072.4$ & 0.763 \\
\hline$\Sigma$ Saturated FA & $26,020.2$ & $26,327.6$ & 400.1 & 0.589 & 0.883 & $26,219.9$ & $26,325.8$ & 609.4 & 0.903 & $25,820.5$ & $26,329.4$ & 518.5 & 0.492 \\
\hline$\Sigma$ Unsaturated FA & $43,409.6$ & $43,332.4$ & 491.3 & 0.912 & 0.285 & $43,990.4$ & 43,862 & 685.7 & 0.896 & $42,828.9$ & $42,802.7$ & 703.9 & 0.979 \\
\hline$\Sigma$ MUFA & $31,589.8$ & $31,418.6$ & 399.1 & 0.763 & 0.723 & $31,887.8$ & $31,540.7$ & 582.5 & 0.676 & $31,291.8$ & $31,296.5$ & 545.8 & 0.995 \\
\hline$\Sigma$ PUFA & $11,819.8$ & $11,913.8$ & 195.8 & 0.736 & 0.047 & $12,102.5$ & $12,321.4$ & 286.4 & 0.593 & $11,537.1$ & $11,506.1$ & 267.1 & 0.935 \\
\hline Omega $3^{5)}$ & 695.8 & 824.6 & 14.9 & $<0.001$ & 0.291 & 714.2 & 839.5 & 23.6 & 0.001 & 677.4 & 809.8 & 18.2 & $<0.001$ \\
\hline Omega 6) & $10,701.3$ & $10,660.6$ & 177.2 & 0.871 & 0.039 & $10,971.3$ & 11,035 & 259.4 & 0.863 & $10,431.4$ & $10,286.2$ & 241.3 & 0.673 \\
\hline Omega 3/0mega 6 & 0.007 & 0.077 & 0.001 & $<0.001$ & 0.102 & 0.065 & 0.076 & 0.0009 & $<0.001$ & 0.065 & 0.079 & 0.001 & $<0.001$ \\
\hline
\end{tabular}

SEM, standard error of the mean; ALA, $\alpha$-linolenic acids, EPA, eicosapentaenoic acid; DHA, docosahexaenoic acid; FA, fatty acids; MUFA, monounsaturated fatty acid; PUFA, polyunsaturated fatty acids.

1) FA below the level of detection are not reported and include the following: C4:0, C6:0, C11:0, C13:0, C:15:1, C17:1, C18:2n6t, C18:2 c9,t11-CLA, C19:0, C21:0, C22:0, C22: C24:1.

2) AURA, unextracted Aurantiochytrium limacinum algae containing $18.0 \mathrm{~g} \mathrm{DHA} / 100 \mathrm{~g}$.

${ }^{3)}$ Effect of treatment and the treatment $\times$ sex interaction reported from the general linear model investigating the effect of treatment on fatty acid profile.

4) Short-chain fatty acids: C4.0 to C8.0; medium-chain fatty acids: C10.0 to C15.1; long-chain fatty acids: C16.0 to C22:6n3.

${ }^{5)}$ The total omega-3 fatty acid composition was calculated as the $\sum$ ( $\alpha$-linolenic acid [C18:3n3]+cis-11,14,17-eicosatrienoic acid [C20:3n3]+EPA [C20:5n3]+DHA [C22:6n3]).

${ }^{6)}$ The total omega-6 fatty-acid composition was calculated as the $\sum$ (linolelaidic acid [C18:2n6trans]+linoleic acid [C18:2n6cis] $+\gamma$-linolenic acid [C18:3n6]+cis-8,11,14-eicosatrienoic acid [C20:3n6]+arachidonic acid [C20:4n6]).

The FA profile of the backfat indicated significantly higher levels of C22:1n9 and DHA and lower levels of C18:1 trans, and C24:0 in the AURA supplemented animals. Overall a significantly higher $\mathrm{n}-3$ content was found in the backfat in addition to an increased n-3:n-6 ratio. Again, the analysis indicated an interaction between sex and treatment for some FAs and as such each sex was analysed separately. Female pigs had significantly lower C18:1 trans and higher DHA. Male pigs had greater levels of C:10, C15:0, C17:0, C22:1n9, and DHA than the control group. Both male and female pigs had an increased $n-3$ concentration and a higher n-3:n-6 ratio.

\section{DISCUSSION}


Supplementation with AURA had no effect on the production parameters or on the measured carcass characteristics, which is generally in agreement with other studies that have fed microalgae [5,10,16,17,21], plant oils [22,23] or fish oils $[6,24]$ to manipulate the FA profile of pork products. Incorporation of $1 \%$ AURA into the diet of pigs with a mean initial weight of $117.1( \pm 13.1) \mathrm{kg}$, for a period of 31 days resulted in significant changes to the FA profile of pork LL and backfat. Pork LL samples from the AURA-fed pigs differed significantly in terms of their EPA and DHA content $(\mathrm{p}<0.0001)$, with treated animals having $62 \%$ and $247 \%$ greater EPA and DHA respectively, than the control group. For the backfat samples, AURA treatment had a significant effect on the level of DHA (with an increase of $218 \%$ compared to controls) but did not lead to an increase in EPA concentration. Overall, pork LL and backfat were enriched to a level of 31.2 and $192.4 \mathrm{mg}$ of DHA/100 g respectively. The effect of dietary supplementation of pigs with AURA had previously been investigated by Moran et al $[17,18]$ over longer periods (117 and 121 days), using animals with lower initial weights (approximately 20 to $30 \mathrm{~kg}$ ) than the current study. In both previous trials, similar increases in LL DHA content (approximately $3 \mathrm{X}$ the control) were observed for pigs supplemented with $0.25 \%$ AURA. In the current study, a similar level of enrichment was achieved after 31 days of supplementation with $1 \%$ AURA. Vossen et al [12], who investigated the effects of dietary supplementation with Schizochytrium (0.3\% to $2.2 \%$ algae) for a period of 45 days found similar levels of enrichment to the current study, reporting 10 to $20 \mathrm{mg}$ of DHA/100 g of pork longissimus thoracis. Sardi et al [11] also used Schizochytrium to supplement the diet of pigs and demonstrated increases in the DHA content of LL from $100 \%$ to $250 \%$. Supplementation with $2.5 \%$ algae for a period of 56 or 28 days led to similar levels of LL enrichment (50 mg and $40 \mathrm{mg} \mathrm{DHA} / 100 \mathrm{~g} \mathrm{LL}$ ). A significantly higher LL DHA content was achieved with supplementation at a level of $5 \%$ for 28 days ( $70 \mathrm{mg} / 100 \mathrm{~g} \mathrm{LL}$ ). These studies indicate that supplementing a higher concentration for a shorter period of time can achieve similar or increased levels of enrichment than longer term supplementation with lower concentrations.

In contrast to the current study, Sardi et al [11] found no increase in the EPA content of LL or backfat. In a seperate study, in which pigs were supplemented with a large dose of 9,400 mg DHA/d, the authors showed a significant increase in both EPA and DHA content of both the LL and backfat [21]. The differences in the EPA content of LL and backfat observed in the current study could be influenced by a number of factors. Firstly, the concentration of ALA, the parent compound of EPA and DHA, differed significantly between the control and treatment LL samples but did not differ in the backfat samples. Smink et al [13] demonstrated that higher concentrations of ALA significantly increased EPA levels in intramuscular fat, but had no effect in the backfat, which may explain the in- creased concentration of EPA in pork LL, but its absence from the backfat in the current study. In addition, following dietary DHA supplementation of pigs, the expression of FA synthesis genes has been shown to increase significantly, in the muscle and liver tissue but not in fat [21]. Secondly, the supplemental levels of ALA and EPA detected in AURA were relatively low (0.02 and $0.23 \mathrm{~g} / 100 \mathrm{~g} \mathrm{DM}$, respectively). As n-3 FAs are preferentially incorporated into phospholipids over triacylglycerol, the low level of supplementation with ALA and EPA might help explain their relative increases in the phospholipid rich muscle as opposed to the triacylglycerol rich backfat [25]. However, it is unlikely that these are the only causes for the increased concentration of EPA in the LL. Vossen et al [12], also observed an increase in the concentration of EPA that could not have come directly from the diet. The authors suggested that higher EPA concentrations in algae supplemented pigs could be attributed to DHA retro-conversion, a minor metabolic pathway which involves one cycle of $\beta$-oxidation [26].

Both previous studies by Moran and colleagues [17,18], indicated that higher levels of AURA supplementation (0.5\%) may affect the sexes differently. While the LL DHA content of both males and females significantly increased relative to the controls, the LL of the males was enriched to a higher degree ( $5 \times$ vs $4.5 \times$ and $4 \times$ vs $2.9 \times$; [17], [18], respectively). In contrast, no treatment $\times$ sex interaction was detected for the pork LL or backfat DHA content in the current study, indicating that the sexes responded similarly under these conditions. However, treatment $\times$ sex interactions were observed for a number of other FAs. In the LL samples of female pigs, the FA composition was significantly different from the control for 17 FAs, while only three significant differences in the male FA profile were observed. In the backfat of female pigs, the only changes detected involved c18:1 trans and DHA, while in male pigs the level of five different FAs differed from the control animals. The FA composition of pigs is mainly influenced by nutrition, however, other factors such as genotype, sex, age and the total amount of carcass fat also impact the individual FA composition [25]. In humans and mice, sex hormones are thought to play a role in the metabolism of n-3 PUFAs [27]. Higher DHA concentrations in the tissues and plasma of women have been attributed to greater levels of DHA synthesis [28]. These studies indicate that the sex hormones may influence FA metabolism and could explain the difference in response to supplementation observed in this study. Overall, EPA and DHA both increased to similar degrees in male and female pigs. In pork LL, an increase in the n-3:n-6 ratio was not observed for the female pigs, likely due to the increase in the level of n-6 FA observed in this group. For the backfat however, no significant increase in n-6 FAs led to similar n-3:n-6 ratios for the both males and females. 


\section{CONCLUSION}

These results indicate that dietary supplementation with $1 \%$ AURA over a 31-day period can increase the FA composition of pork LL and backfat, specifically the DHA, with no major impact on growth performance and carcass traits. Both female and castrated male pigs responded similarly to AURA supplementation in terms of the DHA content of the LL and backfat but differed in terms of a number of other FAs. These results support the use of AURA as an effective, sustainable method by which to increase the $n-3$ content of pork.

\section{CONFLICT OF INTEREST}

Funding for this work was provided by Alltech SARL (France). The author Moran CA and Keegan JD are employees of Alltech which produces and markets ALL-G-RICH, the commercial product used in this study.

\section{ACKNOWLEDGMENTS}

The authors would like to express their gratitude to Ms. Rebecca Timmons (Alltech USA) for her technical input on microalgae and its applications in swine nutrition.

\section{REFERENCES}

1. Beare-Rogers J, Dieffenbacher A, Holm JV. Lexicon of lipid nutrition (IUPAC Technical Report). Pure Appl Chem 2001; 73:685-744.

2. Rossi R, Pastorelli G, Cannata S, Corino C. Recent advances in the use of fatty acids as supplements in pig diets: A review. Anim Feed Sci Technol 2010;162:1-11.

3. Ruxton CHS, Reed SC, Simpson JA, Millington KJ. The health benefits of omega-3 polyunsaturated fatty acids: a review of the evidence. J Hum Nutr Diet 2007;20:275-85.

4. European Food Safety Authority. Scientific opinion on dietary reference values for fats, including saturated fatty acids, polyunsaturated fatty acids, monounsaturated fatty acids, trans fatty acids, and cholesterol. EFSA J 2010;8:1461.

5. Marriott NG, Garrett JE, Sims MD, Abril J. Performance characteristics and fatty acid composition of pigs fed a diet with docosahexaenoic acid. J Muscle Foods 2002;13:265-77.

6. Kjos NP, Skrede A, Øverland M. Effects of dietary fish silage and fish fat on growth performance and sensory quality of growing-finishing pigs. Can J Anim Sci 1999;79:139-47.

7. Haak L, De Smet S, Fremaut D, Van Walleghem K, Raes K. Fatty acid profile and oxidative stability of pork as influenced by duration and time of dietary linseed or fish oil supplementation. J Anim Sci 2008;86:1418-25.

8. Irie M, Sakimoto M. Fat characteristics of pigs fed fish oil containing eicosapentaenoic and docosahexaenoic acids. J
Anim Sci 1992;70:470-7.

9. Gjerlaug-Enger E, Haug A, Gaarder M, et al. Pig feeds rich in rapeseed products and organic selenium increased omega-3 fatty acids and selenium in pork meat and backfat. Food Sci Nutr 2015;3:120-8.

10. Sardi L, Martelli G, Lambertini L, Parisini P, Mordenti A. Effects of a dietary supplement of DHA-rich marine algae on Italian heavy pig production parameters. Livest Sci 2006;103:95-103.

11. Vossen E, Raes K, Van Mullem D, De Smet S. Production of docosahexaenoic acid (DHA) enriched loin and dry cured ham from pigs fed algae: Nutritional and sensory quality. Eur J Lipid Sci Technol 2017;119:1600144.

12.Smink W, Verstegen MWA, Gerrits WJJ. Effect of intake of linoleic acid and a-linolenic acid levels on conversion into long-chain polyunsaturated fatty acids in backfat and in intramuscular fat of growing pigs. J Anim Physiol Anim Nutr (Berl) 2013;97:558-65.

13. Salem N, Eggersdorfer M. Is the world supply of omega-3 fatty acids adequate for optimal human nutrition? Curr Opin Clin Nutr Metab Care 2015;18:147-54.

14. Abril R, Garrett J, Zeller SG, Sander WJ, Mast RW. Safety assessment of DHA-rich microalgae from Schizochytrium sp. Part V: Target animal safety/toxicity study in growing swine. Regul Toxicol Pharmacol 2003;37:73-82.

15. Meadus WJ, Duff P, Uttaro B, et al. Production of docosahexaenoic acid (DHA) enriched bacon. J Agric Food Chem 2010; 58:465-72.

16. Moran CA, Fusconi G, Morlacchini M, Jacques KA. 123 Performance and docosahexaenoic acid (DHA) content in longissimus dorsi and backfat tissues of grow-finish pigs fed diets differing in heterotrophically grown algae content. J Anim Sci 2017; 95(Issue Suppl 2):58.

17. Moran CA, Fusconi G, Morlacchini M, Jacques KA. 124 Docosahexaenoic acid content in longissimus dorsi and backfat tissues of grow-finish pigs fed diets containing $0,0.25$ or $0.5 \%$ heterotrophically grown algae: study 2. J Anim Sci 2017;95 (Issue Suppl 2):58-9.

18. Noblet J, Perez JM. Prediction of digestibility of nutrients and energy values of pig diets from chemical analysis. J Anim Sci 1993;71:3389-98.

19. Noblet J, Fortune H, Shi XS, Dubois S. Prediction of net energy value of feeds for growing pigs. J Anim Sci 1994;72:344-54.

20.O'Fallon JV, Busboom JR, Nelson ML, Gaskins CT. A direct method for fatty acid methyl ester synthesis: Application to wet meat tissues, oils, and feedstuffs. J Anim Sci 2007;85:151121.

21.Meadus WJ, Duff P, Rolland D, et al. Feeding docosahexaenoic acid to pigs reduces blood triglycerides and induces gene expression for fat oxidation. Can J Anim Sci 2011;91:601-12.

22. Kouba M, Enser M, Whittington FM, Nute GR, Wood JD. Effect of a high-linolenic acid diet on lipogenic enzyme activities, fatty acid composition, and meat quality in the 
growing pig. J Anim Sci 2003;81:1967-79.

23. Leskanich CO, Matthews KR, Warkup CC, Noble RC, Hazzledine $M$. The effect of dietary oil containing (n-3) fatty acids on the fatty acid, physicochemical, and organoleptic characteristics of pig meat and fat. J Anim Sci 1997;75:673-83.

24.Bryhni EA, Kjos NP, Ofstad R, Hunt M. Polyunsaturated fat and fish oil in diets for growing-finishing pigs: Effects on fatty acid composition and meat, fat, and sausage quality. Meat Sci 2002;62:1-8.

25. Wood JD, Richardson RI, Nute GR, et al. Effects of fatty acids on meat quality: a review. Meat Sci 2003;66:21-32

26. Sprecher H, Luthria DL, Mohammed BS, Baykousheva SP. Reevaluation of the pathways for the biosynthesis of polyunsaturated fatty acids. J Lipid Res 1995;36:2471-7.

27. Childs CE, Romeu-Nadal M, Burdge GC, Calder PC. Gender differences in the $\mathrm{n}-3$ fatty acid content of tissues. Proc Nutr Soc 2008;67:19-27.

28. Bakewell L, Burdge GC, Calder PC. Polyunsaturated fatty acid concentrations in young men and women consuming their habitual diets. Br J Nutr 2006;96:93-9. 